Canad. J. Math. Vol. 56 (6), 2004 pp. 1339-1342

\title{
Index of Volume 56
}

A 3 Amini, Massoud Locally Compact Pro-C*-Algebras

B 23 Bennett, Michael A., Chris M. Skinner Ternary Diophantine Equations via Galois Representations and Modular Forms

225 Blower, Gordon, Thomas Ransford Complex Uniform Convexity and Riesz Measure

246 Bonnafé, Cédric Éléments unipotents réguliers des sous-groupes de Levi

897 Borwein, David See Jonathan M. Borwein

897 Borwein, Jonathan M., David Borwein, William F. Galway Finding and Excluding $b$-ary Machin-Type Individual Digit Formulae

C 673 Cali, Élie Défaut de semi-stabilité des courbes elliptiques dans le cas non ramifié

1121 Chaumat, Jacques, Anne-Marie Chollet Division par un polynôme hyperbolique

1121 Chollet, Anne-Marie See Jacques Chaumat

D 1145 Daigle, Daniel, Peter Russell On Log (0)-Homology Planes and Weighted Projective Planes

449 Demeter, Ciprian The Best Constants Associated with Some Weak Maximal Inequalities in Ergodic Theory

277 Dostanić, Milutin R. Spectral Properties of the Commutator of Bergman's Projection and the Operator of Multiplication by an Analytic Function

F 472 Fonf, Vladimir P., Libor Vesely Infinite-Dimensional Polyhedrality

1190 Frank, Günter, Xinhou Hua, Rémi Vaillancourt Meromorphic Functions Sharing the Same Zeros and Poles

G 897 Galway, William F. See Jonathan M. Borwein

699 Gaspari, Thierry Bump Functions with Hölder Derivatives

495 Gomi, Yasushi, Iku Nakamura, Ken-ichi Shinoda Coinvariant Algebras of Finite Subgroups of $\operatorname{SL}(3, C)$

716 Guardo, Elena, Adam Van Tuyl Fat Points in $\mathbb{P}^{1} \times \mathbb{P}^{1}$ and Their Hilbert Functions 
1340

H 926 Hadfield, Tom K-Homology of the Rotation Algebras $A_{\theta}$

71 Harper, Malcolm, M. Ram Murty Euclidean Rings of Algebraic Integers

55 Harper, Malcolm $\mathbb{Z}[\sqrt{14}]$ is Euclidean

945 Helminck, Aloysius G., Gerald W. Schwarz Smoothness of Quotients Associated With a Pair of Commuting Involutions

$1228 \mathrm{Ho}$, Nan-Kuo, Chiu-Chu Melissa Liu On the Connectedness of Moduli Spaces of Flat Connections over Compact Surfaces

77 Holmes, Mark, Antal A. Járai, Akira Sakai, Gordon Slade High-Dimensional Graphical Networks of Self-Avoiding Walks

1190 Hua, Xinhou See Günter Frank

I 963 Ishiwata, Satoshi A Berry-Esseen Type Theorem on Nilpotent Covering Graphs

J 77 Járai, Antal A. See Mark Holmes

742 Jiang, Chunlan Similarity Classification of Cowen-Douglas Operators

983 Junge, Marius Fubini's Theorem for Ultraproducts of Noncommutative $L_{p}$-Spaces

K 115 Kenny, Robert Estimates of Hausdorff Dimension for the Non-Wandering Set of an Open Planar Billiard

293 Khomenko, Oleksandr, Volodymyr Mazorchuk Structure of modules induced from simple modules with minimal annihilator

1237 Kishimoto, Akitaka Central Sequence Algebras of a Purely Infinite Simple $C^{*}$-algebra

L 134 Li, Chi-Kwong, Ahmed Ramzi Sourour Linear Operators on Matrix Algebras that Preserve the Numerical Range, Numerical Radius or the States

776 Lim, Yongdo Best Approximation in Riemannian Geodesic Submanifolds of Positive Definite Matrices

1228 Liu, Chiu-Chu Melissa See Nan-Kuo Ho

310 Llibre, Jaume, Dana Schlomiuk The Geometry of Quadratic Differential Systems with a Weak Focus of Third Order

M 1068 Maldeghem, Hendrik Van See Anja Steinbach

529 Martínez-Finkelshtein, A., V. Maymeskul, E. A. Rakhmanov, E. B. Saff Asymptotics for Minimal Discrete Riesz Energy on Curves in $\mathbb{R}^{d}$

1022 Matignon, D., N. Sayari Non-Orientable Surfaces and Dehn Surgeries

529 Maymeskul, V. See A. Martínez-Finkelshtein

293 Mazorchuk, Volodymyr See Oleksandr Khomenko 
344 Miao, Tianxuan Predual of the Multiplier Algebra of $A_{p}(G)$ and Amenability

794 Michel, Laurent Semi-Classical Behavior of the Scattering Amplitude for Trapping Perturbations at Fixed Energy

553 Mohammadalikhani, Ramin Cohomology Ring of Symplectic Quotients by Circle Actions

883 Moran, William See Haryono Tandra

356 Murty, M. Ram, Filip Saidak Non-Abelian Generalizations of the Erdős-Kac Theorem

71 Murty, M. Ram See Malcolm Harper

N 495 Nakamura, Iku See Yasushi Gomi

$566 \mathrm{Ni}$, Yilong Geodesics in a Manifold with Heisenberg Group as Boundary

$590 \mathrm{Ni}$, Yilong The Heat Kernel and Green's Function on a Manifold with Heisenberg Group as Boundary

O 406 Orton, Louisa, Ambrus Pál n Elementary Proof of a Weak Exceptional Zero Conjecture Theta Series, Eisenstein Series and Poincaré Series over Function Fields 373 Orton, Louisa An Elementary Proof of a Weak Exceptional Zero Conjecture

P 406 Pál, Ambrus See Louisa Orton

612 Pál, Ambrus Solvable Points on Projective Algebraic Curves

1259 Paterson, Alan L. T. The Fourier Algebra for Locally Compact Groupoids

825 Penot, Jean-Paul Differentiability Properties of Optimal Value Functions

168 Pogge, James Todd On a Certain Residual Spectrum of $\mathrm{Sp}_{8}$

R 529 Rakhmanov, E. A. See A. Martínez-Finkelshtein

225 Ransford, Thomas See Gordon Blower

431 Rosenblatt, Joseph, Michael Taylor Group Actions and Singular Martingales II, The Recognition Problem

1034 Rouleux, Michel Semi-classical Integrability,Hyperbolic Flows and the Birkhoff Normal Form

843 Ruan, Zhong-Jin Type Decomposition and the Rectangular AFD Property for $W^{*}$-TRO's

1145 Russell, Peter See Daniel Daigle

S 529 Saff, E. B. See A. Martínez-Finkelshtein

356 Saidak, Filip See M. Ram Murty

194 Saikia, A. Selmer Groups of Elliptic Curves with Complex Multiplication 
1342

77 Sakai, Akira See Mark Holmes

1022 Sayari, N. See D. Matignon

310 Schlomiuk, Dana See Jaume Llibre

209 Schmuland, Byron, Wei Sun A Central Limit Theorem and Law of the Iterated Logarithm for a Random Field with Exponential Decay of Correlations

871 Schocker, Manfred Lie Elements and Knuth Relations

945 Schwarz, Gerald W. See Aloysius G. Helminck

1290 Scull, Laura Equivariant Formality for Actions of Torus Groups

495 Shinoda, Ken-ichi See Yasushi Gomi

23 Skinner, Chris M. See Michael A. Bennett

77 Slade, Gordon See Mark Holmes

638 Śniatycki, Jedrzej Multisymplectic Reduction for Proper Actions

134 Sourour, Ahmed Ramzi See Chi-Kwong Li

1068 Steinbach, Anja, Hendrik Van Maldeghem Regular Embeddings of Generalized Hexagons

209 Sun, Wei See Byron Schmuland

T 883 Tandra, Haryono, William Moran Kirillov Theory for a Class of Discrete Nilpotent Groups

655 Tao, Xiangxing, Henggeng Wang On the Neumann Problem for the Schrödinger Equations with Singular Potentials in Lipschitz Domains

431 Taylor, Michael See Joseph Rosenblatt

1094 Thomas, Hugh Cycle-Level Intersection Theory for Toric Varieties

716 Tuyl, Adam Van See Elena Guardo

V 1190 Vaillancourt, Rémi See Günter Frank

472 Veselý, Libor See Vladimir P. Fonf

W 655 Wang, Henggeng See Xiangxing Tao

Z 1308 Zhao, Jianqiang Variations of Mixed Hodge Structures of Multiple Polylogarithms 